\title{
Quasi-Lovász extensions on bounded chains
}

\author{
Miguel Couceiro and Jean-Luc Marichal \\ 1 LAMSADE - CNRS, UniversitÃC) Paris-Dauphine \\ Place du MarÃ (C) chal de Lattre de Tassigny \\ 75775 Paris Cedex 16, France \\ miguel.couceiro@dauphine.fr \\ ${ }^{2}$ University of Luxembourg, Mathematics Research Unit \\ 6, rue Richard Coudenhove-Kalergi \\ L-1359 Luxembourg, G.-D. Luxembourg \\ jean-luc.marichal@uni.lu
}

\begin{abstract}
We study quasi-Lovász extensions as mappings $f: C^{n} \rightarrow \mathbb{R}$ defined on a nonempty bounded chain $C$, and which can be factorized as $f\left(x_{1}, \ldots, x_{n}\right)=L\left(\varphi\left(x_{1}\right), \ldots, \varphi\left(x_{n}\right)\right)$, where $L$ is the Lovász extension of a pseudo-Boolean function $\psi:\{0,1\}^{n} \rightarrow \mathbb{R}$ and $\varphi: C \rightarrow \mathbb{R}$ is an order-preserving function.

We axiomatize these mappings by natural extensions to properties considered in the authors' previous work. Our motivation is rooted in decision making under uncertainty: such quasi-Lovász extensions subsume overall preference functionals associated with discrete Choquet integrals whose variables take values on an ordinal scale $C$ and are transformed by a given utility function $\varphi: C \rightarrow \mathbb{R}$.

Furthermore, we make some remarks on possible lattice-based variants and bipolar extensions to be considered in an upcoming contribution by the authors.
\end{abstract}

Keywords: Aggregation function, discrete Choquet integral, Lovász extension, quasi-Lovász extension, comonotonic modularity.

MSC classes: 39B22, 39B72 (Primary) 26B35 (Secondary)

\section{Introduction and preliminary results}

The discrete Choquet integral has been widely investigated in aggregation theory due to its many applications, for instance, in decision making (see the edited book [8]). A convenient way to introduce the discrete Choquet integral is via the concept of Lovász extension.

\subsection{Lovász extensions and the discrete Choquet integral}

Let $C$ be a chain under a linear order $\leqslant$ or, equivalently, under a minimum $\wedge$ or a maximum $\vee$; for instance, $\mathbb{B}:=\{0,1\}$ with the usual ordering given by 
$0<1$. Given a permutation $\sigma \in S_{n}$, where $S_{n}$ denotes the set of permutations on $[n]=\{1, \ldots, n\}$, we define

$$
C_{\sigma}^{n}:=\left\{\mathbf{x}=\left(x_{1}, \ldots, x_{n}\right) \in C^{n}: x_{\sigma(1)} \leqslant \cdots \leqslant x_{\sigma(n)}\right\} .
$$

For every $A \subseteq[n]$, we denote by $\mathbf{1}_{A}$ the $n$-tuple whose $i$-th component is 1 if $i \in A$ and is 0 otherwise. For instance, $\mathbf{1}_{\emptyset}$ is the constant 0 tuple, denoted $\mathbf{0}$, whereas $\mathbf{1}_{[n]}$ is the constant 1 tuple.

Consider an $n$-place pseudo-Boolean function, i.e. a function $\psi: \mathbb{B}^{n} \rightarrow \mathbb{R}$, and define the set function $v_{\psi}: 2^{[n]} \rightarrow \mathbb{R}$ by $v_{\psi}(A)=\psi\left(\mathbf{1}_{A}\right)$ for every $A \subseteq[n]$. Hammer and Rudeanu [9] showed that such a function has a unique representation as a multilinear polynomial of $n$ variables

$$
\psi(\mathbf{x})=\sum_{A \subseteq[n]} a_{\psi}(A) \prod_{i \in A} x_{i}
$$

where the set function $a_{\psi}: 2^{[n]} \rightarrow \mathbb{R}$, called the Möbius transform of $v_{\psi}$, is defined by

$$
a_{\psi}(A)=\sum_{B \subseteq A}(-1)^{|A|-|B|} v_{\psi}(B) .
$$

The Lovász extension of a pseudo-Boolean function $\psi: \mathbb{B}^{n} \rightarrow \mathbb{R}$ is the function $L_{\psi}: \mathbb{R}^{n} \rightarrow \mathbb{R}$ whose restriction to each subdomain $\mathbb{R}_{\sigma}^{n}\left(\sigma \in S_{n}\right)$ is the unique affine function which agrees with $\psi$ at the $n+1$ vertices of the $n$-simplex $[0,1]^{n} \cap \mathbb{R}_{\sigma}^{n}$ (see $[10,11]$ ). We then have $\left.L_{\psi}\right|_{\mathbb{B}^{n}}=\psi$.

It can be shown (see $[7, \S 5.4 .2]$ ) that the Lovász extension of a pseudo-Boolean function $\psi: \mathbb{B}^{n} \rightarrow \mathbb{R}$ is the continuous function

$$
L_{\psi}(\mathbf{x})=\sum_{A \subseteq[n]} a_{\psi}(A) \bigwedge_{i \in A} x_{i}, \quad \mathbf{x} \in \mathbb{R}^{n} .
$$

Its restriction to $\mathbb{R}_{\sigma}^{n}$ is the affine function

$$
L_{\psi}(\mathbf{x})=\psi(\mathbf{0})+\sum_{i \in[n]} x_{\sigma(i)}\left(v_{\psi}\left(A_{\sigma}^{\uparrow}(i)\right)-v_{\psi}\left(A_{\sigma}^{\uparrow}(i+1)\right)\right), \quad \mathbf{x} \in \mathbb{R}_{\sigma}^{n},
$$

or equivalently,

$$
L_{\psi}(\mathbf{x})=\psi(\mathbf{0})+\sum_{i \in[n]} x_{\sigma(i)}\left(L_{\psi}\left(\mathbf{1}_{A_{\sigma}^{\uparrow}(i)}\right)-L_{\psi}\left(\mathbf{1}_{A_{\sigma}^{\uparrow}(i+1)}\right)\right), \quad \mathbf{x} \in \mathbb{R}_{\sigma}^{n},
$$

where $A_{\sigma}^{\uparrow}(i)=\{\sigma(i), \ldots, \sigma(n)\}$, with the convention that $A_{\sigma}^{\uparrow}(n+1)=\varnothing$. Indeed, for any $k \in[n+1]$, both sides of each of the equations (2) and (3) agree at $\mathbf{x}=\mathbf{1}_{A_{\sigma}^{\uparrow}(k)}$.

It is noteworthy that $L_{\psi}$ can also be represented by

$$
L_{\psi}(\mathbf{x})=\psi(\mathbf{0})+\sum_{i \in[n]} x_{\sigma(i)}\left(L_{\psi}\left(-\mathbf{1}_{A_{\sigma}^{\downarrow}(i-1)}\right)-L_{\psi}\left(-\mathbf{1}_{A_{\sigma}^{\downarrow}(i)}\right)\right), \quad \mathbf{x} \in \mathbb{R}_{\sigma}^{n},
$$


where $A_{\sigma}^{\downarrow}(i)=\{\sigma(1), \ldots, \sigma(i)\}$, with the convention that $A_{\sigma}^{\downarrow}(0)=\varnothing$. Indeed, for any $k \in[n+1]$, by (3) we have

$$
L_{\psi}\left(-\mathbf{1}_{A_{\sigma}^{\downarrow}(k-1)}\right)=\psi(\mathbf{0})+L_{\psi}\left(\mathbf{1}_{A_{\sigma}^{\uparrow}(k)}\right)-L_{\psi}\left(\mathbf{1}_{A_{\sigma}^{\uparrow}(1)}\right) .
$$

A function $f: \mathbb{R}^{n} \rightarrow \mathbb{R}$ is said to be a Lovász extension if there is a pseudoBoolean function $\psi: \mathbb{B}^{n} \rightarrow \mathbb{R}$ such that $f=L_{\psi}$.

An $n$-place Choquet integral is a nondecreasing Lovász extension $L_{\psi}: \mathbb{R}^{n} \rightarrow$ $\mathbb{R}$ such that $L_{\psi}(\mathbf{0})=0$. It is easy to see that a Lovász extension $L: \mathbb{R}^{n} \rightarrow \mathbb{R}$ is an $n$-place Choquet integral if and only if its underlying pseudo-Boolean function $\psi=\left.L\right|_{\mathbb{B}^{n}}$ is nondecreasing and vanishes at the origin (see $[7, \S 5.4]$ ).

\subsection{Quasi-Lovász extensions on bounded chains}

A generalization of the notion of Lovász extension of a pseudo-Boolean function was introduced in [4] and called "quasi-Lovász extension". In this paper we extend this notion to the following concept.

Let $C$ be a bounded chain under the usual operations $\wedge$ and $\vee$, and with least and greatest elements 0 and 1 , respectively. We make no notational distinction between $0,1 \in C$ and $0,1 \in \mathbb{R}$; this notational abuse will not give raise to ambiguities.

We say that a function $f: C^{n} \rightarrow \mathbb{R}$ is a quasi-Lovász extension if there is a Lovász extension $L: \mathbb{R}^{n} \rightarrow \mathbb{R}$ and an order-preserving mapping $\varphi: C \rightarrow \mathbb{R}$ such that $f$ can be factorized into the composition

$$
f\left(x_{1}, \ldots, x_{n}\right)=L\left(\varphi\left(x_{1}\right), \ldots, \varphi\left(x_{n}\right)\right) .
$$

For such a $\varphi: C \rightarrow \mathbb{R}$ we set $\varphi(C):=\{\varphi(x): x \in C\}$ that is contained in some real interval $I$. To simplify our exposition we will assume that $\varphi(0)=0$ and that $\varphi(C) \subseteq I=[0, a]$, for $a=\varphi(1)$ (shift by $\varphi(0)$ if necessary). Also, for every $f: C^{n} \rightarrow \mathbb{R}$, we denote by $f_{0}$ the function $f_{0}(\mathbf{x}):=f(\mathbf{x})-f(\mathbf{0})$.

Such an aggregation function is used in decision under uncertainty, where $\varphi$ is a utility function and $f$ an overall preference functional. It is also used in multicriteria decision making where the criteria are commensurate (i.e., expressed in a common scale). For a recent reference, see Bouyssou et al. [1].

\section{Axiomatizations and representations of quasi-Lovász extensions on bounded chains}

We now provide an axiomatization of quasi-Lovász extensions on bounded chains in terms of comonotonic modularity and an ordinal variant of homogeneity, as well as provide a complete description of all possible factorizations (into a composition of a Lovász extension with an order-preserving unary mapping) when such a factorization exists. 


\subsection{Comonotonically modular and separable functions}

Recall that a function $f: C^{n} \rightarrow \mathbb{R}$ is said to be modular (or a valuation) if

$$
f(\mathbf{x})+f\left(\mathbf{x}^{\prime}\right)=f\left(\mathbf{x} \wedge \mathbf{x}^{\prime}\right)+f\left(\mathbf{x} \vee \mathbf{x}^{\prime}\right)
$$

for every $\mathbf{x}, \mathbf{x}^{\prime} \in C^{n}$, and where $\mathbf{x} \wedge \mathbf{x}^{\prime}$ (resp. $\mathbf{x} \vee \mathbf{x}^{\prime}$ ) denotes the $n$-tuple whose $i$ th component is $x_{i} \wedge x_{i}^{\prime}=\min \left(x_{i}, x_{i}^{\prime}\right)$ (resp. $x_{i} \vee x_{i}^{\prime}=\max \left(x_{i}, x_{i}^{\prime}\right)$ ). It was proved (see Topkis [12, Thm 3.3]) that a function $f: C^{n} \rightarrow \mathbb{R}$ is modular if and only if it is separable, that is, there exist $n$ functions $f_{i}: C \rightarrow \mathbb{R}, i \in[n]$, such that $f=\sum_{i \in[n]} f_{i} \cdot{ }^{3}$ In particular, any 1-place function $f: C \rightarrow \mathbb{R}$ is modular.

We say that a function $f: C^{n} \rightarrow \mathbb{R}$ is comonotonically modular if (5) holds for every $\sigma \in S_{n}$ and $\mathbf{x}, \mathbf{x}^{\prime} \in C_{\sigma}^{n}$.

Fact 1 A function $f$ is comonotonically modular if and only if so is the function $f_{0}=f-f(\mathbf{0})$.

Theorem 2. A function $f: C^{n} \rightarrow \mathbb{R}$ is comonotonically modular if and only if one or, equivalently, both of the conditions hold:

(i) there exists a function $g: C^{n} \rightarrow \mathbb{R}$ such that, for every $\sigma \in S_{n}$ and every $\mathbf{x} \in C_{\sigma}^{n}$

$$
f(\mathbf{x})=g(\mathbf{0})+\sum_{i \in[n]}\left(g\left(x_{\sigma(i)} \wedge \mathbf{1}_{A_{\sigma}^{\uparrow}(i)}\right)-g\left(x_{\sigma(i)} \wedge \mathbf{1}_{A_{\sigma}^{\uparrow}(i+1)}\right)\right) .
$$

In this case, we can choose $g=f$.

(ii) $f$ is comonotonically separable, that is, for every $\sigma \in S_{n}$, there exist functions $f_{i}^{\sigma}: C \rightarrow \mathbb{R}, i \in[n]$, such that

$$
f(\mathbf{x})=\sum_{i=1}^{n} f_{i}^{\sigma}\left(x_{\sigma(i)}\right)=\sum_{i=1}^{n} f_{\sigma^{-1}(i)}^{\sigma}\left(x_{i}\right), \quad \mathbf{x} \in C_{\sigma}^{n} .
$$

In this case, we can choose $f_{n}^{\sigma}\left(x_{\sigma(n)}\right)=f\left(x_{\sigma(n)} \wedge \mathbf{1}_{\{\sigma(n)\}}\right)$, and $f_{i}^{\sigma}\left(x_{\sigma(i)}\right)=$ $f\left(x_{\sigma(i)} \wedge \mathbf{1}_{A_{\sigma}^{\uparrow}(i)}\right)-f\left(x_{\sigma(i)} \wedge \mathbf{1}_{A_{\sigma}^{\uparrow}(i+1)}\right)$, for $i \in[n-1]$.

Proof. It is easy to verify that $(i) \Rightarrow(i i)$. Moreover, each $f_{\sigma^{-1}(i)}^{\sigma}$ is clearly modular and hence comonotonically modular. Since the class of comonotonically modular functions is closed under addition, we have that (ii) is sufficient. Thus, to complete the proof, it is enough to show that every comonotonically modular function $f$ satisfies $(i)$.

Let $\sigma \in S_{n}$ and $\mathbf{x} \in C_{\sigma}^{n}$. By comonotonic modularity, for every $i \in[n-1]$ we have

$$
f\left(x_{\sigma(i)} \wedge \mathbf{1}_{A_{\sigma}^{\uparrow}(i)}\right)+f\left(\mathbf{x}_{A_{\sigma}^{\downarrow}(i)}^{0}\right)=f\left(x_{\sigma(i)} \wedge \mathbf{1}_{A_{\sigma}^{\uparrow}(i+1)}\right)+f\left(\mathbf{x}_{A_{\sigma}^{\downarrow}(i-1)}^{0}\right),
$$

that is,

$$
f\left(\mathbf{x}_{A_{\sigma}^{\downarrow}(i-1)}^{0}\right)=\left(f\left(x_{\sigma(i)} \wedge \mathbf{1}_{A_{\sigma}^{\uparrow}(i)}\right)-f\left(x_{\sigma(i)} \wedge \mathbf{1}_{A_{\sigma}^{\uparrow}(i+1)}\right)\right)+f\left(\mathbf{x}_{A_{\sigma}^{\downarrow}(i)}^{0}\right) .
$$

By using (7) for $i=1, \ldots, n-1$, we obtain (6) with $g=f$.

\footnotetext{
${ }^{3}$ This result still holds in the more general framework where $f$ is defined on a product of chains.
} 


\subsection{Axiomatizations of quasi-Lovász extensions}

To axiomatize the class of quasi-Lovász extensions, we will also make use of the following generalization of homogeneity. We say that $f: C^{n} \rightarrow \mathbb{R}$ is weakly homogeneous if there exists an order-preserving function $\varphi: C \rightarrow \mathbb{R}$ satisfying $\varphi(0)=0$ such that $f\left(x \wedge \mathbf{1}_{A}\right)=\varphi(x) f\left(\mathbf{1}_{A}\right)$ for every $x \in C$ and every $A \subseteq[n]$. Note that every weakly homogeneous function $f$ satisfies $f(\mathbf{0})=0$.

The following two results are variants of Lemma 2 and Proposition 3 in [4]. and their proofs follow in complete analogy.

Lemma 3. For every quasi-Lovász extension $f: C^{n} \rightarrow \mathbb{R}, f=L \circ \varphi$, we have

$$
f_{0}\left(x \wedge \mathbf{1}_{A}\right)=\varphi(x) L_{0}\left(\mathbf{1}_{A}\right), \quad x \in C, A \subseteq[n] .
$$

Proposition 4. Let $f: C^{n} \rightarrow \mathbb{R}$ be a nonconstant quasi-Lovász extension, $f=$ $L \circ \varphi$. Then the following conditions are equivalent.

(i) $f_{0}$ is weakly homogeneous.

(ii) There exists $A \subseteq[n]$ such that $f_{0}\left(\mathbf{1}_{A}\right) \neq 0$.

(iii) $\varphi(1) \neq 0$.

In this case we have $f_{0}\left(x \wedge \mathbf{1}_{A}\right)=\frac{\varphi(x)}{\varphi(1)} f_{0}\left(\mathbf{1}_{A}\right)$ for every $x \in C$ and every $A \subseteq[n]$.

We can now provide axiomatizations of the class of quasi-Lovász extensions defined on bounded chains. The proof follows the same steps as in the proof of Theorem 14 in [4].

Theorem 5. Let $f: C^{n} \rightarrow \mathbb{R}$ be a nonconstant function. Then, the following assertions are equivalent.

(i) $f$ is a quasi-Lovász extension and there exists $A \subseteq[n]$ such that $f_{0}\left(\mathbf{1}_{A}\right) \neq 0$.

(ii) $f$ is comonotonically modular and $f_{0}$ is weakly homogeneous.

(iii) There is an order-preserving function $\varphi_{f}: C \rightarrow \mathbb{R}$ satisfying $\varphi_{f}(0)=0$ and $\varphi_{f}(1)=1$ such that $f=L_{\left.f\right|_{\{0,1\} n}} \circ \varphi_{f}$.

Proof. Let us prove that $(i) \Rightarrow(i i)$. By definition, we have $f=L \circ \varphi$, where $L: \mathbb{R}^{n} \rightarrow \mathbb{R}$ is a Lovász extension and $\varphi: C \rightarrow \mathbb{R}$ is an order-preserving function satisfying $\varphi(0)=0$. By Proposition $4, f_{0}$ is weakly homogeneous. Moreover, by (3) and (8) we have that, for every $\sigma \in S_{n}$ and every $\mathbf{x} \in C_{\sigma}^{n}$,

$$
\begin{aligned}
f(\mathbf{x}) & =f(\mathbf{0})+\sum_{i \in[n]} \varphi\left(x_{\sigma(i)}\right)\left(L_{0}\left(\mathbf{1}_{A_{\sigma}^{\uparrow}(i)}\right)-L_{0}\left(\mathbf{1}_{A_{\sigma}^{\uparrow}(i+1)}\right)\right) \\
& =f(\mathbf{0})+\sum_{i \in[n]}\left(f\left(x_{\sigma(i)} \mathbf{1}_{A_{\sigma}^{\uparrow}(i)}\right)-f\left(x_{\sigma(i)} \mathbf{1}_{A_{\sigma}^{\uparrow}(i+1)}\right)\right) .
\end{aligned}
$$

Theorem 2 then shows that $f$ is comonotonically modular.

Let us prove that $(i i) \Rightarrow($ iii). Since $f$ is comonotonically modular, by Theorem 2 it follows that, for every $\sigma \in S_{n}$ and every $\mathbf{x} \in C_{\sigma}^{n}$,

$$
f(\mathbf{x})=f(\mathbf{0})+\sum_{i \in[n]}\left(f\left(x_{\sigma(i)} \wedge \mathbf{1}_{A_{\sigma}^{\uparrow}(i)}\right)-f\left(x_{\sigma(i)} \wedge \mathbf{1}_{A_{\sigma}^{\uparrow}(i+1)}\right)\right),
$$


and, since $f_{0}$ is weakly homogeneous,

$$
f(\mathbf{x})=f(\mathbf{0})+\sum_{i \in[n]} \varphi_{f}\left(x_{\sigma(i)}\right)\left(f\left(\mathbf{1}_{A_{\sigma}^{\uparrow}(i)}\right)-f\left(\mathbf{1}_{A_{\sigma}^{\uparrow}(i+1)}\right)\right)
$$

for some order-preserving function $\varphi_{f}: C \rightarrow \mathbb{R}$ satisfying $\varphi_{f}(0)=0$. By (3), we then obtain $f=L_{\left.f\right|_{\{0,1\}^{n}}} \circ \varphi_{f}$. Finally, by (9) we have that, for every $A \subseteq[n]$,

$$
f_{0}\left(\mathbf{1}_{A}\right)=\varphi_{f}(1) f_{0}\left(\mathbf{1}_{A}\right) .
$$

Since there exists $A \subseteq[n]$ such that $f_{0}\left(\mathbf{1}_{A}\right) \neq 0$ (for otherwise, we would have $f_{0} \equiv 0$ by $\left.(9)\right)$, we obtain $\varphi_{f}(1)=1$.

The implication $(i i i) \Rightarrow(i)$ follows from Proposition 4.

Remark 6. It is noteworthy that the class of quasi-polynomial functions on bounded chains [2] (or, more generally, on bounded distributive lattices [3]) is likewise axiomatizable in terms of comonotonic modularity by considering a lattice variant of weak homogeneity [6], namely: a function $f: C^{n} \rightarrow \mathbb{R}$ is said to be weakly $\wedge$-homogeneous if there exists an order-preserving function $\varphi: C \rightarrow \mathbb{R}$ such that $f\left(x \wedge \mathbf{1}_{A}\right)=\varphi(x) \wedge f\left(\mathbf{1}_{A}\right)$ for every $x \in C$ and every $A \subseteq[n]$.

\subsection{Factorizations of quasi-Lovász extensions}

We now describe all possible factorizations of $f$ into compositions of Lovász extensions with order-preserving functions.

Theorem 7. Let $f: C^{n} \rightarrow \mathbb{R}$ be a quasi-Lovász extension, $f=L \circ \varphi$. Then there exists $A^{*} \subseteq[n]$ such that $f_{0}\left(\mathbf{1}_{A^{*}}\right) \neq 0$ if and only if there exists a $>0$ such that $\varphi=a \varphi_{f}$ and $L_{0}=\frac{1}{a}\left(L_{\left.f\right|_{\mathbb{B}}}\right)_{0}$.

Proof. (Sufficiency) We have $f_{0}=L_{0} \circ \varphi=\left(L_{\left.f\right|_{\{0,1\}^{n}}}\right)_{0} \circ \varphi_{f}$, and by Theorem 5 we see that the conditions are sufficient.

(Necessity) By Proposition 4, we have

$$
\frac{\varphi(x)}{\varphi(1)}=\frac{f_{0}\left(x \wedge \mathbf{1}_{A^{*}}\right)}{f_{0}\left(\mathbf{1}_{A^{*}}\right)}=\varphi_{f}(x) .
$$

We then have $\varphi=a \varphi_{f}$ for some $a>0$. Moreover, for every $\mathbf{x} \in\{0,1\}^{n}$, we have

$$
\begin{aligned}
\left(L_{\left.f\right|_{\{0,1\}^{n}}}\right)_{0}(\mathbf{x}) & =\left(\left(L_{\left.f\right|_{\{0,1\}^{n}}}\right)_{0} \circ \varphi_{f}\right)(\mathbf{x})=f_{0}(\mathbf{x})=\left(L_{0} \circ \varphi\right)(\mathbf{x}) \\
& =a\left(L_{0} \circ \varphi_{f}\right)(\mathbf{x})=a L_{0}(\mathbf{x}) .
\end{aligned}
$$

Since a Lovász extension is uniquely determined by its values on $\{0,1\}^{n} \subseteq \mathbb{R}$, we have $\left(L_{\left.f\right|_{\{0,1\}^{n}}}\right)_{0}=a L_{0}$. 


\section{Concluding remarks and directions for further research}

We have axiomatized the class of quasi-Lovász extensions, considered in the wider setting of functions $f: C^{n} \rightarrow \mathbb{R}$ defined on a bounded chain $C$, thus partially generalizing the results presented in the proceedings of IPMU2012 [5]. It remains now to consider the symmetric variant of the notion of quasi-Lovász extension defined on bipolar scales $C=C^{+} \cup C^{-}$where $C^{+}$is a bounded chain and $C^{-}$is its "negative" copy.

\section{Acknowledgments}

This research is supported by the internal research project F1R-MTH-PUL12RDO2 of the University of Luxembourg.

\section{References}

1. D. Bouyssou, D. Dubois, H. Prade, and M. Pirlot, editors. Decision-Making Process - Concepts and Methods. ISTE/John Wiley, London, 2009.

2. M. Couceiro and J.-L. Marichal. Axiomatizations of quasi-polynomial functions on bounded chains. Aeq. Math., 78(1-2):195-213, 2009.

3. M. Couceiro and J.-L. Marichal. Quasi-polynomial functions over bounded distributive lattices. Aeq. Math., 80(3):319-334, 2010.

4. M. Couceiro and J.-L. Marichal. Axiomatizations of quasi-Lovász extensions of pseudo-Boolean functions, Aequationes Mathematicae 82 (2011) 213-231.

5. M. Couceiro and J.-L. Marichal. Quasi-LovÃ $j$ sz extensions and their symmetric counterparts. Information Processing and Management of Uncertainty in Knowledge-Based Systems, Communications in Computer and Information Science, vol. 300, Springer-Verlag, 178-187, 2012.

6. M. Couceiro and J.-L. Marichal. Discrete integrals based on comonotonic modularity, Axioms 2:3 (2013) 390-403.

7. M. Grabisch, J.-L. Marichal, R. Mesiar, and E. Pap. Aggregation functions. Encyclopedia of Mathematics and its Applications 127. Cambridge University Press, Cambridge, UK, 2009.

8. M. Grabisch, T. Murofushi, and M. Sugeno, editors. Fuzzy measures and integrals - Theory and applications, volume 40 of Studies in Fuzziness and Soft Computing. Physica-Verlag, Heidelberg, 2000.

9. P. Hammer and S. Rudeanu. Boolean methods in operations research and related areas. Berlin-Heidelberg-New York: Springer-Verlag, 1968.

10. L. Lovász. Submodular functions and convexity. In Mathematical programming, 11th int. Symp., Bonn 1982, 235-25\%. 1983.

11. I. Singer. Extensions of functions of 0-1 variables and applications to combinatorial optimization. Numer. Funct. Anal. Optimization, 7:23-62, 1984.

12. D. M. Topkis. Minimizing a submodular function on a lattice. Operations Research, 26(2):305-321, 1978. 